\title{
BOUNDARY LAYER COLLAPSES DESCRIBED BY THE TWO-DIMENSIONAL INTERMEDIATE LONG-WAVE EQUATION
}

\author{
Joseph O. Oloo ${ }^{1,2}$ \\ \& \\ Victor I. Shrira ${ }^{1}$ \\ ${ }^{1}$ School of Computing and Mathematics, Keele University, \\ ST5 5BG Staffordshire, United Kingdom \\ 2 The Catholic University of Eastern Africa, P.O Box 62157 \\ 00200, Nairobi, Kenya. \\ j.o.oloo@keele.ac.uk \\ v.i.shrira@keele.ac.uk
}

September 9, 2020

\begin{abstract}
We study the nonlinear dynamics of localized perturbations of a confined generic boundary-layer shear flow in the framework of the essentially two-dimensional generalization of the intermediate long-wave equation (2d-ILW). The 2d-ILW equation was originally derived to describe nonlinear evolution of the boundary layer perturbations in a fluid confined between two parallel planes. The distance between the planes is characterized by a dimensionless parameter $D$. In the limit of large and small $D$, the 2d-ILW equation respectively tends to the $2 \mathrm{~d}$ Benjamin-Ono and $2 \mathrm{~d}$ ZakharovKuznetsov equations. We show that the localized initial perturbations of any given shape collapse, i.e., blow up in finite time and form a point singularity, if the Hamiltonian is negative, which occurs if the perturbation amplitude exceeds a certain threshold specific for each particular shape of the initial perturbation. For the axisymmetric Gaussian and Lorentzian initial perturbations of amplitude $a$ and width $\sigma$, we derive explicit nonlinear neutral stability curves that separate the domains of perturbation collapse and decay on the plane $(a, \sigma)$ for various values of $D$. The amplitude threshold
\end{abstract}


$a$ increases as $D$ and $\sigma$ decrease and tends to infinity at $D \rightarrow 0$. The 2 d-ILW equation also admits steady axisymmetric solitary wave solutions whose Hamiltonian is always negative; they collapse for all $D$ except $D=0$, they have no amplitude threshold. But the equation itself has not been proved for small $D$. Direct numerical simulations of the 2d-ILW equation with Gaussian and Lorentzian initial conditions show that initial perturbations with an amplitude exceeding the found threshold collapse in a self-similar manner, while perturbations with a below-threshold amplitude decay. 


\section{Introduction}

Boundary layers are ubiquitous in nature and in the engineering context. Understanding the evolution of boundary layers perturbations is crucial for controlling the laminar-turbulent transition. The huge literature on this topic accumulated over more than a century is mostly concerned with studies of linear instabilities, i.e., situations where infinitesimal perturbations grow and the role of nonlinearity is primarily in modifying this growth (see, e.g., [1]).

Here, we focus on nonlinear growth of weakly decaying modes. The basic model we consider is the $2 \mathrm{~d}$ intermediate long-wave equation (2d-ILW), which was derived in [2], (see Eqs. 35,36,52). This equation is a member of a broader family of two-dimensional nonlinear evolution equations derived in [2, and it describes the evolution of weakly nonlinear longwave perturbations of a generic boundary layer in a homogeneous fluid confined between two parallel planes for a wide range of Reynolds numbers. Its one dimensional version was derived by Joseph in [3] and independently in [4] for long waves in stratified inviscid fluid of finite depth in situations where the stratification is confined to a thin layer. It has an infinite number of integrals of motions and has soliton and multi-soliton solutions (see, e.g., [5], [6], [7]). The equation has a dimensionless parameter $D$ characterizing the overall depth of the fluid. In the limits of large and small $D$, it respectively reduces to the integrable Benjamin-Ono and Korteweg-de Vries equations.

In contrast to its well studied $1 d$ reduction, the $2 \mathrm{~d}-\mathrm{ILW}$ equation has never been studied, beyond numerically finding its localized $2 \mathrm{~d}$ axisymmetric solitary wave solutions in [2]. In the linear limit, solutions of the 2d-ILW equation are superpositions of harmonic waves satisfying a dispersion relation between the frequency $\omega$ and wave vector $\boldsymbol{k}=\left\{k_{x}, k_{y}\right\}$,

$$
\omega=-k_{x}|\boldsymbol{k}| \operatorname{coth}(|\boldsymbol{k}| D)
$$

where $D$ is the dimensionless distance between two parallel plates confining the boundary layer. It is easy to see that as $D \rightarrow \infty$ this relation reduces to the dispersion relation of the linearized 2d Benjamin-Ono equation, i.e., $\omega=-k_{x}|\boldsymbol{k}|$ in [8]. In the opposite limit $D \rightarrow 0$, we obtain $\omega=-k_{x}\left(1 / D-D|\boldsymbol{k}|^{2}\right)$, which (after a Galilean transformation) corresponds to the dispersion relation of the linearized 2d Zakharov-Kuznetsov (ZK) equation [9]. Correspondingly, the 2d-ILW equation reduces to the $2 \mathrm{~d}$ Benjamin-Ono equation for large $D$ and after the same Galilean transformation to the $2 \mathrm{~d}-\mathrm{ZK}$ equation for small $D$.

In 1995, Dyachenko and Kusnetsov showed [10] that the essentially 2d Benjamin-Ono equation obtained by one of us in 1989 [8] describes collapses of initially localized 2d perturbations. Also in 1995, Pelinovsky and Shrira [11] using the Whitham's adiabatic approach obtained an explicit description of the collapse in the framework of the $2 \mathrm{~d}$ Benjamin-Ono equation [11. In contrast, solitary wave solutions of the $2 \mathrm{~d}-\mathrm{ZK}$ equation localized in two dimensions are considered stable and noncollapsing [12]. Here, we consider the open question of what is the result of the evolution of initially localized perturbations in the framework of the 2d-ILW equation. Can this equation describe collapses, and if yes, then under what conditions?

This paper is organized as follows. In section $\S 2$, we set up the physical model of a generic confined boundary layer and explain the assumptions underlying the asymptotic derivation of 
the 2 d-ILW equation. In section $\S 3$, we describe its basic properties. In section $\S 4$ following [10], we argue that collapses in the 2d-ILW equation occur whenever the Hamiltonian is negative and unbounded and based on this find the explicit domains of collapse and decay in the parameter space for Gaussian and Lorentzian initial perturbations. In section $\S 5$, we describe the numerical simulation of the 2d-ILW equation and present an example of collapse. This example demonstrates the self-similar character of the collapse in the 2d-ILW equation. We also compare the direct numerical simulations of the 2d-ILW equation with the analytic self-similar solution and show that the results agree well. Finally, in section $\S 6$, we summarize our results and discuss the main open questions.

\section{The Model}

\subsection{Boundary layer between two parallel planes}

We consider a typical unidirectional shear flow in a boundary layer confined between two parallel planes separated by a distance $D$. In the Cartesian frame with the origin at the upper boundary with $z, 0 \leq z \leq D$, directed downwards with $x$ and $y$ directed streamwise and spanwise, respectively (Fig 1). The directions are labeled vertical and horizontal only for convenience. Without loss of generality, we assume the unperturbed unidirectional boundary layer velocity $U(z)$ is adjacent to the top boundary and localized with characteristic thickness $d \ll D$. We also assume that the flow profile $U(z)$ has no inflection points. In this setting, the nonlinear evolution of long-wave perturbations with comparable scales in the $x$ and $y$ directions is described by the 2d-ILW equation derived from the Navier-Stokes equations in [2],

$$
A_{\tau}-\alpha_{1} A A_{x}-\beta_{1} \hat{G}\left[A_{x}\right]=0,
$$

where $A(x, y, \tau)$ is the amplitude of the streamwise velocity component of the perturbation, $\tau$ is the slow time, $\alpha_{1}=U^{\prime}(0), \beta_{1}=U^{2}(0) / U^{\prime}(0)$ and the integral operator $\hat{G}[\psi]$ is,

$$
\hat{G}[\psi(\boldsymbol{r})]=\frac{1}{4 \pi^{2}} \int_{-\infty}^{+\infty} \int_{-\infty}^{+\infty} k \operatorname{coth}(k D) \psi\left(\boldsymbol{r}_{1}\right) e^{\left(i \boldsymbol{k}\left(\boldsymbol{r}-\boldsymbol{r}_{1}\right)\right)} \mathrm{d} \boldsymbol{k} \mathrm{d} \boldsymbol{r}_{1}
$$

where $\boldsymbol{r}=\{x, y\}, k=|\boldsymbol{k}|=\left(k_{x}^{2}+k_{y}^{2}\right)^{1 / 2}$. We note that the streamwise perturbation of velocity $u(x, y, z, t)$ in the leading order is expressed in terms of the amplitude $A(x, y, t)$ as

$$
\begin{gathered}
u(x, y, z, t)=-(f(x, y, z) * A(x, y, \tau)) \cdot U^{\prime}(z), \\
f(x, y, z)=\frac{1}{4 \pi^{2}} \int_{\infty}^{\infty}\left[\frac{\sinh k(\varepsilon z+D)}{\sinh (k D)}\right] e^{(-i \boldsymbol{k} r))} \mathrm{d} \boldsymbol{k},
\end{gathered}
$$

where $f * g$ is the convolution in the $\boldsymbol{r}$ space and $\varepsilon \ll 1$ is the small parameter of nonlinearity defined as the ratio of the maximum streamwise velocity of the perturbation to the maximum velocity of the basic flow. Other velocity components can also be expressed in terms of $A(x, y, \tau)$ (see [2] for details). 


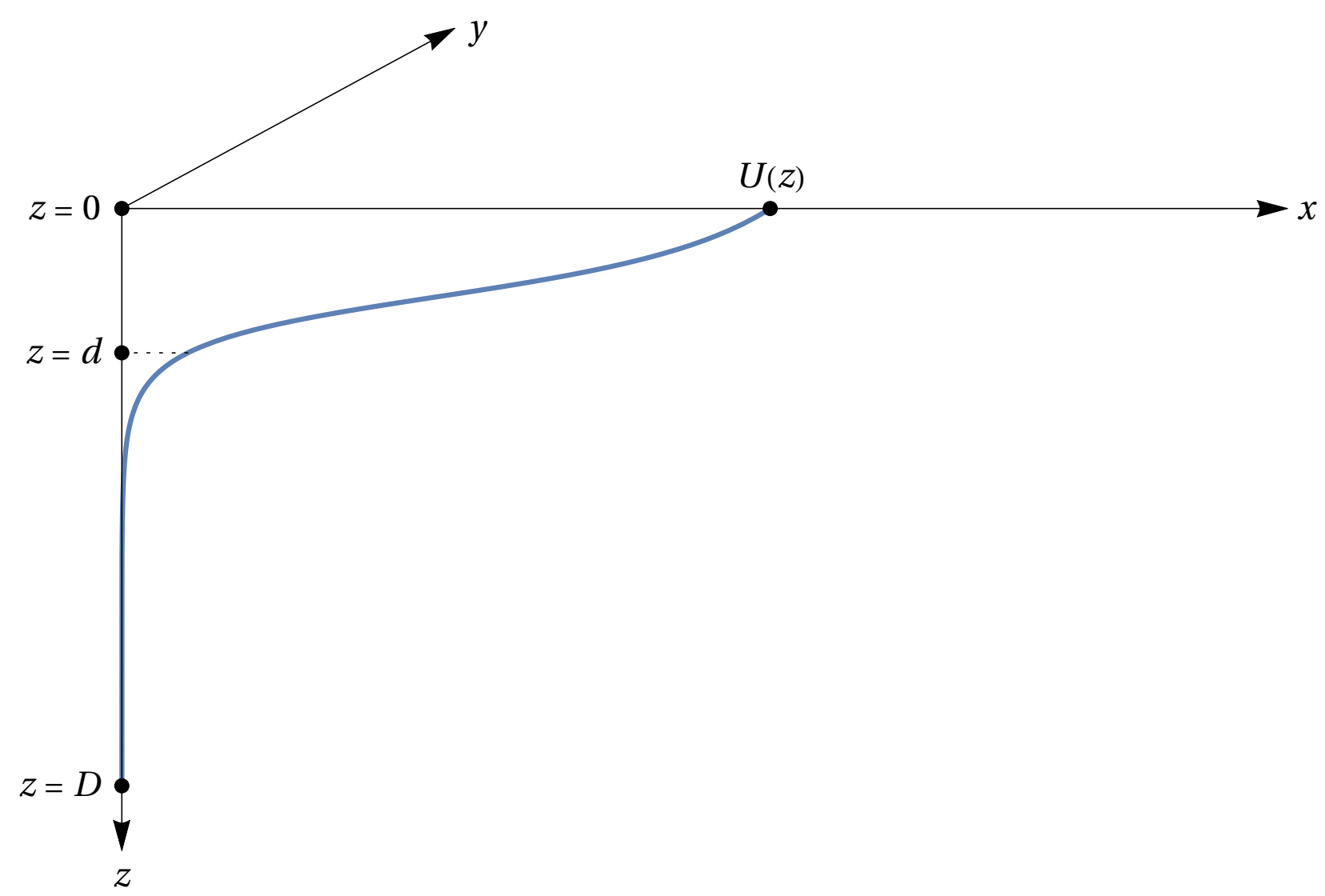

Figure 1: Qualitative form of the geometry of a typical confined boundary-layer profile with the shear localized in a thin boundary layer of thickness $d$ and confined by a second boundary at a distance $D$ : just for definiteness, the boundary layer is shown for a fluid flow with a free boundary. For the typical situations with no slip boundary (e.g., wind tunnels), this picture is obtained after a Galilean transformation: $U(0)$ should be set to zero, and $U(D)$ then becomes the free stream velocity.

Re-scaling the variables by setting $d=1$, we bring the 2d-ILW equation to a form containing a single dimensionless parameter $D$ in the kernel of $\hat{G}$ given by (2)

$$
A_{\tau}+A A_{x}-\hat{G}\left[A_{x}\right]=0 .
$$

This form of the 2d-ILW equation is the mathematical basis of our study.

\section{Basic properties of the 2d-ILW equation}

The 1d-ILW equation is a well-studied weakly nonlinear pseudodifferential equation governing the evolution of long internal waves in a stratified fluid of finite depth (see, e.g., [3], [4]). In contrast, the 2d-ILW equation has not been studied beyond a brief analysis of its 
steady-state solutions in 2] where it was found that the 2d-ILW equation has axisymmetric solitary wave solutions. For waves propagating in an arbitrary direction, 2d-ILW equation (3) reduces to the 1d-ILW equation and, as is known, has an infinite set of integrals of motion, multisoliton solutions, and other properties typical of integrable systems (see, e.g., [5], [6]. [7], [13]).

A notable feature of the 1d-ILW equation is that in the shallow-water limit (i.e., as $D \rightarrow 0$ ), it passes into the classical Korteweg-de Vries equation, while in the deep-water limit $(D \rightarrow \infty)$, it reduces to the Benjamin-Ono equation. Similarly, the 2d-ILW equation reduces to the $2 \mathrm{~d}$ Benjamin-Ono equation in the limit $k D \gg 1$. It is easy to see that in the opposite limit $k D \ll 1$, the leading terms of the Taylor expansion of the function $k \operatorname{coth}(k D)$ in the kernel of $\hat{G}$ have the form $\frac{1}{D}\left(1+\frac{k^{2} D^{2}}{3}\right)$, and $\hat{G}$ consequently becomes the 2 d Laplace operator $\Delta=\partial_{x x}^{2}+\partial_{y y}^{2}$. Hence, for $k D \ll 1$ the 2 d-ILW equation tends to the $2 \mathrm{~d}-\mathrm{ZK}$ equation, which was obtained for describing low-frequency ion-acoustic waves in magnetized plasma [9], but was later found to arise in many other physical contexts [14], 15], [16], [17] and [18]. We note that strictly speaking, it is not legitimate to consider the 2d-ILW equation in the ZK limit. Although the equation itself does admit such a consideration and indeed reduces to the ZK equation, the asymptotic procedure used to derive the $2 \mathrm{~d}$-ILW equation breaks down for small $D$.

We can write 2d-ILW equation (3) in the Hamiltonian form as

$$
A_{\tau}=\partial_{x}\left[\hat{G}[A]-\frac{1}{2} A^{2}\right]=\partial_{x} \frac{\delta H}{\delta A},
$$

where the Hamiltonian $H$ has two constituents $I_{1}$ and $I_{2}$,

$$
H=\frac{1}{2} I_{1}-\frac{1}{6} I_{2}, \quad I_{1}=\int A \hat{G}[A] \mathrm{d} \boldsymbol{r}, \quad I_{2}=\int A^{3} \mathrm{~d} \boldsymbol{r}, \quad(\mathrm{d} \boldsymbol{r} \equiv d x d y) .
$$

In addition to the Hamiltonian, 2d-ILW equation (3) conserves the streamwise and spanwise components of the "momentum" $\boldsymbol{P}$ and the mass flux $M$,

$$
P_{x}=\frac{1}{2} \iint A^{2} \mathrm{~d} x \mathrm{~d} y, \quad P_{y}=\frac{1}{2} \iint A \phi_{y} \mathrm{~d} x \mathrm{~d} y, \quad M=\iint A \mathrm{~d} x \mathrm{~d} y .
$$

where $\left(\phi_{x} \equiv A\right)$. The way these integrals depend on the perturbation parameters allows inferring the existence of collapses for certain initial conditions and describing the domain of collapsing initial conditions for particular chosen classes of initial conditions in the next section.

\section{Collapses in the 2d-ILW equation: Domain of col- lapses in the parameter space}

\subsection{Domain of collapses in the $a-\sigma$ space in the generic case}

Dyachenko and Kusnetsov showed the existence of collapses in the 2d Benjamin-Ono equation [10], which is a particular limit of the 2d-ILW equation. Here, we extend their approach 
to the 2d-ILW equation.

The plane soliton solution of the 2d-ILW equation is stable in the framework of the 1dILW equation but is unstable under transverse modulations in the $2 \mathrm{~d}$-ILW equation that we study. In contrast to the $2 \mathrm{~d}$ Benjamin-Ono equation, for which the transverse stability of plane wave solutions was thoroughly studied in [19], [20], we here skip this part of the analysis because the transverse instability of the plane soliton is obviously manifested in numerical simulations of the 2d-ILW equation and we initially know the scaling of the instability growth rate.

The key result of Dyachenko and Kusnetsov is that the existence of collapses is related to the unboundedness of the Hamiltonian from below as the streamwise momentum $P_{x}$ is kept constant [10]. For any chosen localized initial conditions, a sufficient condition for the collapse to occur is that the Hamiltonian must be negative. The reasoning in [10] is entirely applicable in the case of the $2 \mathrm{~d}$-ILW equation. Using direct numerical simulations of the 2d-ILW equation (which we describe below), we verify that this criterion indeed predicts the occurrence of collapse. Moreover, this criterion allows obtaining a good initial idea how the fate (i.e., collapse or decay) of an initial perturbation depends on its parameters. To outline these dependences, we examine a few simple distributions, Gaussian and Lorentzian pulses,

$$
A_{G}(x, y)=a e^{-\left(x^{2}+y^{2}\right) / 2 \sigma^{2}}, \quad A_{L}(x, y)=\frac{a}{1+4\left(x^{2} / \sigma^{2}+y^{2} / \sigma^{2}\right)} .
$$

These initial perturbations are fully characterized by just two parameters: the amplitude $a$ and characteristic half-width $\sigma$, which we call the width for brevity.

It is easy to see that in the Hamiltonian $H$ given by (5), both its constituent integrals, the dispersion $I_{1}$ and the nonlinear $I_{2}$, can be expressed in terms of amplitude $a$ and the perturbation width $\sigma$. Rescaling the variables

$$
\tilde{x}=\frac{x}{\sigma}, \quad \tilde{y}=\frac{y}{\sigma}, \quad A(\tilde{x}, \tilde{y})=a \mathcal{A}, \quad \mathcal{A}_{G}=e^{-\left(\tilde{x}^{2}+\tilde{y}^{2}\right) / 2}, \quad \mathcal{A}_{L}=\frac{1}{1+4\left(\tilde{x}^{2}+\tilde{y}^{2}\right)} .
$$

we re-write our integrals as,

$$
I_{1}=a^{2} \sigma \tilde{I}_{1}, \quad \tilde{I}_{1}=\iint \mathcal{A} \hat{G}[\mathcal{A}] \mathrm{d} \tilde{x} \mathrm{~d} \tilde{y}, \quad I_{2}=a^{3} \sigma^{2} \tilde{I}_{2}, \quad \tilde{I}_{2}=\iint \mathcal{A}^{3} \mathrm{~d} \tilde{x} \mathrm{~d} \tilde{y}
$$

where $\tilde{I}_{1}$ depends implicitly on $\sigma$ via the integral operator kernel $\operatorname{coth}\left(\frac{k D}{\sigma}\right)$ and $\tilde{I}_{2}$ is a constant that is evaluated analytically or numerically. For the Gaussian initial conditions, $\tilde{I}_{2}=\frac{2}{3} \pi$. The integral $\tilde{I}_{1}$ cannot be evaluated analytically and is treated numerically for the whole range of $D$ and $\sigma$.

If the dispersion and nonlinear contributions in the Hamiltonian exactly balance, i.e., $3 I_{1}-I_{2}=0$, then the Hamiltonian vanishes and thus yields a nonlinear "neutral curve" separating the domains of collapse and decay on the plane $(a, \sigma)$,

$$
a^{2} \sigma\left(3 \tilde{I}_{1}-a \sigma \tilde{I}_{2}\right)=0
$$


The relation between the amplitude threshold $a_{t h r}$ and perturbation width $\sigma$ prescribed by Eq. (8) is,

$$
a_{t h r}(\sigma)=\frac{3 \tilde{I}_{1}}{\tilde{I}_{2} \sigma}
$$

To better understand this relation, we expand $\operatorname{coth}\left(\frac{k D}{\sigma}\right)$ for $\sigma \gg 1$ and $\sigma \ll 1$. Retaining the first two terms in the Maclaurin series of $\operatorname{coth} s$, with $s=\frac{k D}{\sigma} \ll 1$ we obtain, $\frac{1}{s}+\frac{1}{3} s-\ldots$. Similarly, for short waves $\sigma \ll 1$, i.e., for $s \gg 1$, coth $s \approx 1$ with an exponentially small discrepancy (see, e.g., [21]).

\subsection{Domain of collapses in the ZK limit}

We note that the transition from the 2d-ILW equation to the $2 \mathrm{~d}$-ZK equation is not entirely smooth, because in the leading order, we have a large constant translation term $1 / s$, which can be eliminated by switching to the moving coordinate frame. Writing the equation in the Hamiltonian form, we scale the integrals of motion using parameters of the initial conditions, the amplitude $a$ and width $\sigma$. It is hence easy to see that the Hamiltonian $H$ in the ZK limit consists of three terms (the constant translational term $I_{3}$, the dispersion term, $I_{4}$, and the nonlinear term $I_{2}$ ), which can be expressed in terms of the amplitude $a$ and perturbation width $\sigma$,

$$
\begin{gathered}
I_{3}=3 a^{2} \sigma^{2} \tilde{I}_{3} / D, \quad \tilde{I}_{3}=\iint \mathcal{A}^{2} d \tilde{x} d \tilde{y}, \\
I_{4}=D a^{2} \tilde{I}_{4}, \quad \tilde{I}_{4}=\iint \mathcal{A} \hat{G}_{1}[\mathcal{A}] d \tilde{x} d \tilde{y}, \\
I_{2}=a^{3} \sigma^{2} \tilde{I}_{2}, \quad \tilde{I}_{2}=\iint \mathcal{A}^{3} d \tilde{x} d \tilde{y} .
\end{gathered}
$$

We recall that in the ZK limit, the dispersion operator $\hat{G}_{1}$ in the Fourier space is $k^{2} \equiv$ $|\boldsymbol{k}|^{2}=k_{x}^{2}+k_{y}^{2}$. The factor $\tilde{I}_{3}$ is evaluated analytically or numerically; for the Gaussian initial conditions, its exact value is $\pi$. The integral $\tilde{I}_{4}$ is treated numerically and yields a constant.

If the dispersion and nonlinear contributions in the Hamiltonian exactly balance, i.e., $I_{3}+I_{4}-I_{2}=0$, then the Hamiltonian vanishes and thus yields a nonlinear neutral curve separating the domains of collapse and decay on the plane $(a, \sigma)$,

$$
a^{2}\left(\frac{3 \sigma^{2} \tilde{I}_{3}}{D}+D \tilde{I}_{4}-a \sigma^{2} \tilde{I}_{2}\right)=0 .
$$

Therefore, in the long-wave limit, the nonlinear neutral curve specifying the amplitude threshold $a_{t h r}(\sigma)$ corresponding to the ZK equation tends to a constant independent of $\sigma$,

$$
a_{t h r}=\frac{3 \tilde{I}_{3}}{D \tilde{I}_{2}}+\frac{D \tilde{I}_{4}}{\sigma^{2} \tilde{I}_{2}}
$$


We note that asymptotic expression (11) for the threshold amplitude corresponding to the 2d-ZK equation agrees well with the expression obtained from the 2d-ILW equation (see Eq. (9D). Therefore, the amplitude threshold in this particular limit depends primarily on $D$ (it tends to infinity as $1 / D$ as $D \rightarrow 0$ ), while the dependence on the width $\sigma$ vanishes. In the $\sigma \gg 1$ limit, the amplitude threshold ceases to depend on $\sigma$.

In the opposite limit $(\sigma \ll D)$, the neutral curve is the hyperbola,

$$
a_{t h r}=\frac{3 \hat{I}_{1}}{\hat{I}_{2} \sigma} .
$$

We obtained the neutral curves for the intermediate values of $D$ by evaluating $I_{1}$ and $I_{2}$ numerically for axisymmetric Gaussian and Lorentzian initial pulses. Plots of the results are shown in Fig. (2). Perturbations with $a$ and $\sigma$ above the neutral curve invariably collapse, and those with $a$ and $\sigma$ below the curve decay. We note that the curves for the Gaussian and Lorentzian initial conditions are very similar; the quantitative discrepancy is rather small. We emphasize two features of these plots:

1. the amplitude threshold increases monotonically as $D$ decreases and tends to infinity as $D$ tends to zero, and

2. for any $D \neq 0$, the amplitude threshold increases monotonically as $\sigma$ decreases.

In the limit of small $D$ corresponding to the ZK equation, the threshold amplitude stops depending on $\sigma$ and tends to a large $O(1 / D)$ constant. Because the 2d-ILW equation was derived under a weak-nonlinearity assumption, a substantial portion of the collapse domain for small $D$ is outside the applicability domain of the equation. We note that here we considered whether a given initial perturbation lump of amplitude $a$ and width $\sigma$ would collapse assuming that $a$ and $\sigma$ are independent. The conclusion is that the threshold amplitude tends to zero as the width tends to infinity. But if we allow $\sigma$ to depend on

$a$, then at first glance, we obtain a different picture. For example, if we choose the lump solitary wave solutions reported in [2], as our initial condition, then their Hamiltonian is always negative, and all such initial conditions hence collapse for any initial amplitude. Hence, there is no amplitude threshold for this class of initial perturbations. In fact, there is no contradiction with the conclusions obtained for initial distributions with a simple shape: the picture merely becomes somewhat distorted if $\sigma=\sigma(a)$. For the lump solitons in [2], as $\sigma \rightarrow \infty$ as $a \rightarrow 0$, i.e., for infinitely long initial perturbations of whatever shape, the amplitude threshold tends to zero.

\section{Self-similar solution and numerical simulation of col- lapses}

\subsection{Self-similar solution}

It is natural to expect a self-similar behaviour of the solution in the vicinity of the singularity. A self-similar solution of the $2 \mathrm{~d}$ Benjamin-Ono equation was proposed in [10]. In this section, 
we show that the same logic applies to the 2d-ILW equation and find a self-similar solution of (1).

We consider the spatial and temporal dependence of a collapsing perturbation in a certain vicinity of the blowup singularity occurring at $\boldsymbol{r}=\boldsymbol{r}_{0}\left\{x_{0}, y_{0}\right\}$. We introduce the time $\check{\tau}$ to the singularity and the distances $\check{x}=x-x_{0}, \check{y}=y-y_{0}$ to singularity. We seek a self-similar solution of the form

$$
A(\boldsymbol{r}, \check{\tau})=\check{\tau}^{\lambda} g(\boldsymbol{\xi}), \quad \boldsymbol{\xi}=\frac{\boldsymbol{r}}{\check{\tau}^{\mu}} .
$$

Substituting this ansatz in the 2d-ILW equation of form (3) and ensuring that the equation remains invariant, we can easily show that $\lambda=-\frac{1}{2}$ and $\mu=\frac{1}{2}$. Therefore,

$$
u(\boldsymbol{r}, \check{\tau})=\check{\tau}^{-1 / 2} g(\boldsymbol{\xi}), \quad \boldsymbol{\xi}=\check{\tau}^{-1 / 2} \boldsymbol{r}
$$

where $g(\boldsymbol{\xi})$ is given by,

$$
\frac{\boldsymbol{\xi} g}{2}+\frac{g^{2}}{2}+\hat{G}[g]=0
$$

We have thus obtained an explicit self-similar solution of the 2d-ILW equation predicting the singularity $1 / \sqrt{\tau_{0}-\tau}$ in time and the shrinking spatial distribution given by $g(\boldsymbol{\xi})$. Its width tends to zero as $\sqrt{\tau_{0}-\tau}$. This is the self-similar solution of the $2 \mathrm{~d}$ Benjamin-Ono equation obtained in [10] (up to a typo in the time-dependence exponent there). Although our equation for $g$ specifying the shape of the distribution has a simpler form (because we integrated our equation once), it has the same solution.

Hence, we obtain the same self-similar solution for an essentially different equation because the asymptotic forms of the two different equations coincide near the singularity. Here, the shrinking spatial scale of the solution implies an infinite increase of the dominant wave numbers and hence $k D \rightarrow \infty$. Therefore, in the $2 \mathrm{~d}$-ILW equation, the vicinity of the singularity is always (for $D \neq 0$ ) governed by the $2 \mathrm{~d}$ Benjamin-Ono equation. The possible existence of other interesting self-similar regimes for the 2d-ILW equation corresponding to intermediate asymptotic forms not yet identified remains open.

In our numerical experiments, we also considered the axisymmetric "ground solitons" of 2d-ILW found in [2] as the initial conditions. The Hamiltonian for such distributions is negative, they hence collapse, and there is no amplitude threshold. This holds for any $D \neq 0$. As $D \rightarrow 0$ the 2 d-ILW ground soliton becomes the $2 \mathrm{~d}-\mathrm{ZK}$ ground soliton. Although the Hamiltonian of the ground soliton remains negative, the Hamiltonian becomes bounded in the 2d-ZK limit (see [12], 22]), which suggests that the 2d-ZK ground soliton is stable. There are several numerical studies of the $2 \mathrm{~d}-\mathrm{ZK}$ equation where its ground solitons were used as the initial conditions and were found to be stable (see, e.g., [23]).

We do not discuss the $2 \mathrm{~d}-\mathrm{ZK}$ equation further here, because the 2d-ILW equation, as mentioned, becomes inapplicable in this limit.

\subsection{Numerical Simulation}

To simulate the 2d-ILW equation numerically for localized initial perturbations, we use the pseudospectral method (see, e.g., [24], 25]). This method with periodic boundary conditions 
uses efficient fast Fourier transform routines to handle dependences on $x$ and $y$ and the classic fourth-order Runge-Kutta method for the time evolution.

In our context, it was found to be optimal to use a large rectangular box of length $256 \pi$ and width $64 \pi$. This choice provides sufficient domain for the spatial decay of the simulated localized perturbations and also allows the perturbation sufficient time to move in the streamwise direction during the evolution. To resolve the rapidly growing, increasingly localized amplitudes, we used $4096 \times 1024$ grid points. We numerically solved 2d-ILW equation (3) in the moving coordinate frame. It is convenient to present our evolution equation in the flux-conservation form $A_{\tau}+F_{x}=0$, where the flux $F=-\hat{G}[A]+\frac{1}{2} A^{2}$. The integral operator $\hat{G}[A]$ was treated in the Fourier space, and the nonlinear terms were considered in the physical space on collocation points with the "two-third de-aliasing rule" [[24]. The accuracy of the simulations was controlled by ensuring that the integrals of motion (5) and (6) remain constant with an error not exceeding $10^{-4}$.

\subsection{Evolution scenarios}

After obtaining nonlinear neutral curves separating the collapse and decay domains in section \$4, we verified the findings by numerical simulations of the evolution of axisymmetric Gaussian pulse initial conditions (7). Choosing the initial perturbations slightly above and slightly below a curve, we confirmed that the initial perturbations with amplitudes exceeding the threshold indeed collapse, i.e., such perturbations evolve into a short-lived pattern that tends to a localized self-similar solution with a point singularity. Initial conditions below the threshold decay, although a temporary transient growth, sometimes substantial, can also occur.

In Figures (3) and (4), we illustrate a typical evolution of a collapsing pulse for a localized initial condition. A typical time dependence of a collapsing pulse amplitude simulated for an axisymmetric Gaussian initial condition and $D=4$ is shown in Figure (3). We note that the amplitude increases slowly for most of its evolution and the abrupt growth occurs just immediately before the singularity. This figure also gives a good idea of how surprisingly well self-similar solution (14) of the $2 \mathrm{~d}$ Benjamin-Ono equation captures the evolution governed by the 2 d-ILW equation. A complementary view of the evolution is shown in Figure (4), with a sequence of snapshots of a collapsing pulse

Here, we emphasize two notable features. Although the initial condition is perfectly axisymmetric, the emerging pattern is not: at the pedestal, the pulse radiates in two distinguished directions, and the resulting pattern resembles "hair-pin" or "lambda" vortices routinely observed in wind tunnels (see, e.g.,[26]). The top of the pulse evolves in an axisymmetric self-similar manner tending to a point singularity at the end. Asymmetric initial conditions also have these properties. 


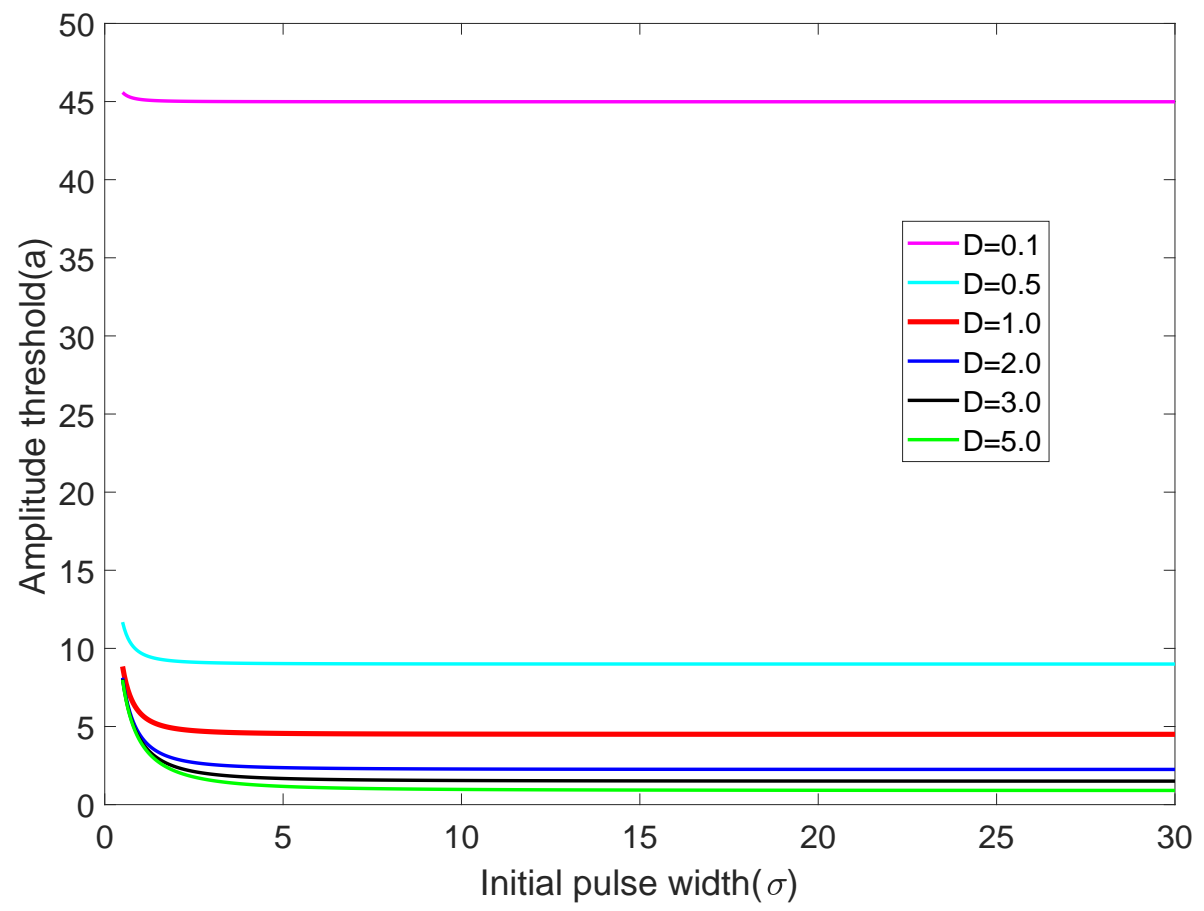

(a)

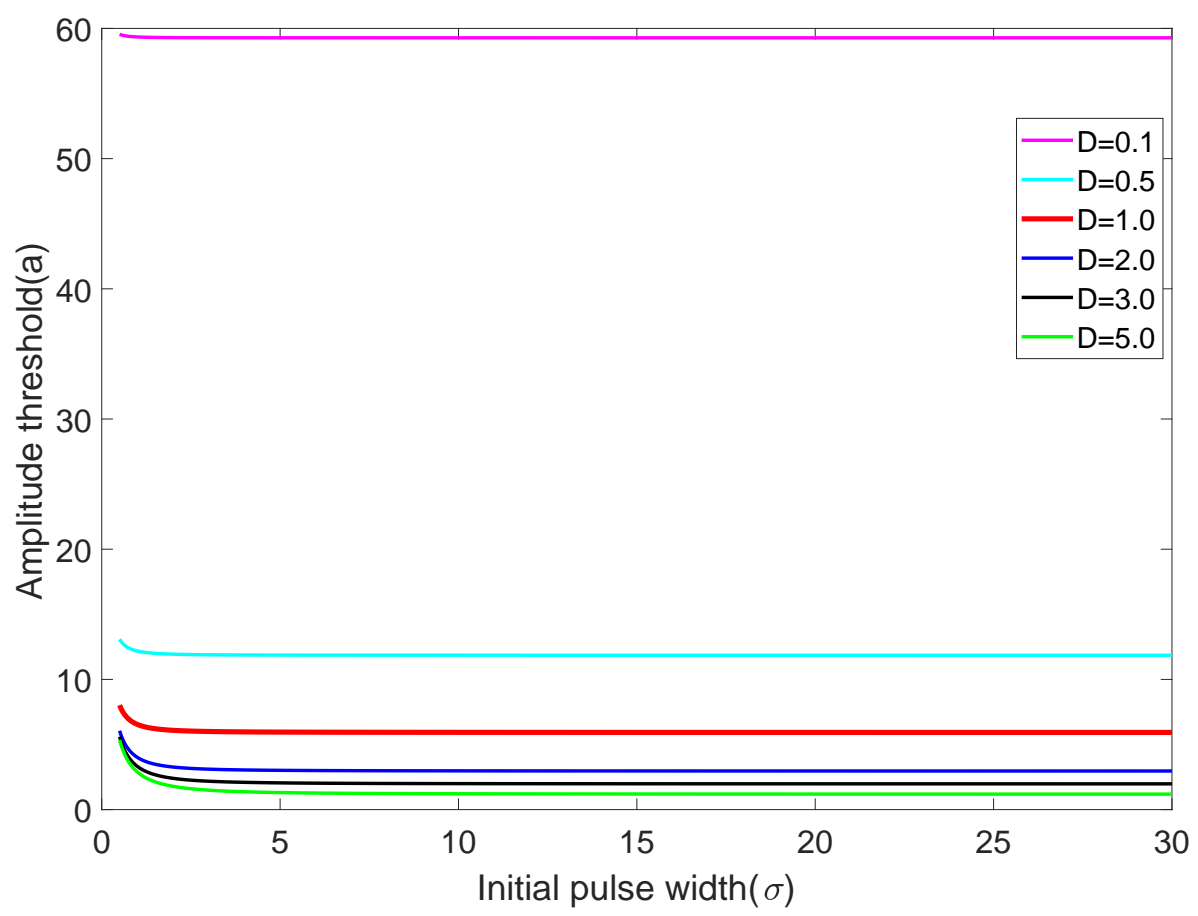

(b)

Figure 2: 'Neutral curves' separating the domains of collapse and decay for different values of $D(D=0.1,0.5,1,2,3,5)$ for: (a) the Gaussian and (b) the Lorentzian initial conditions. 


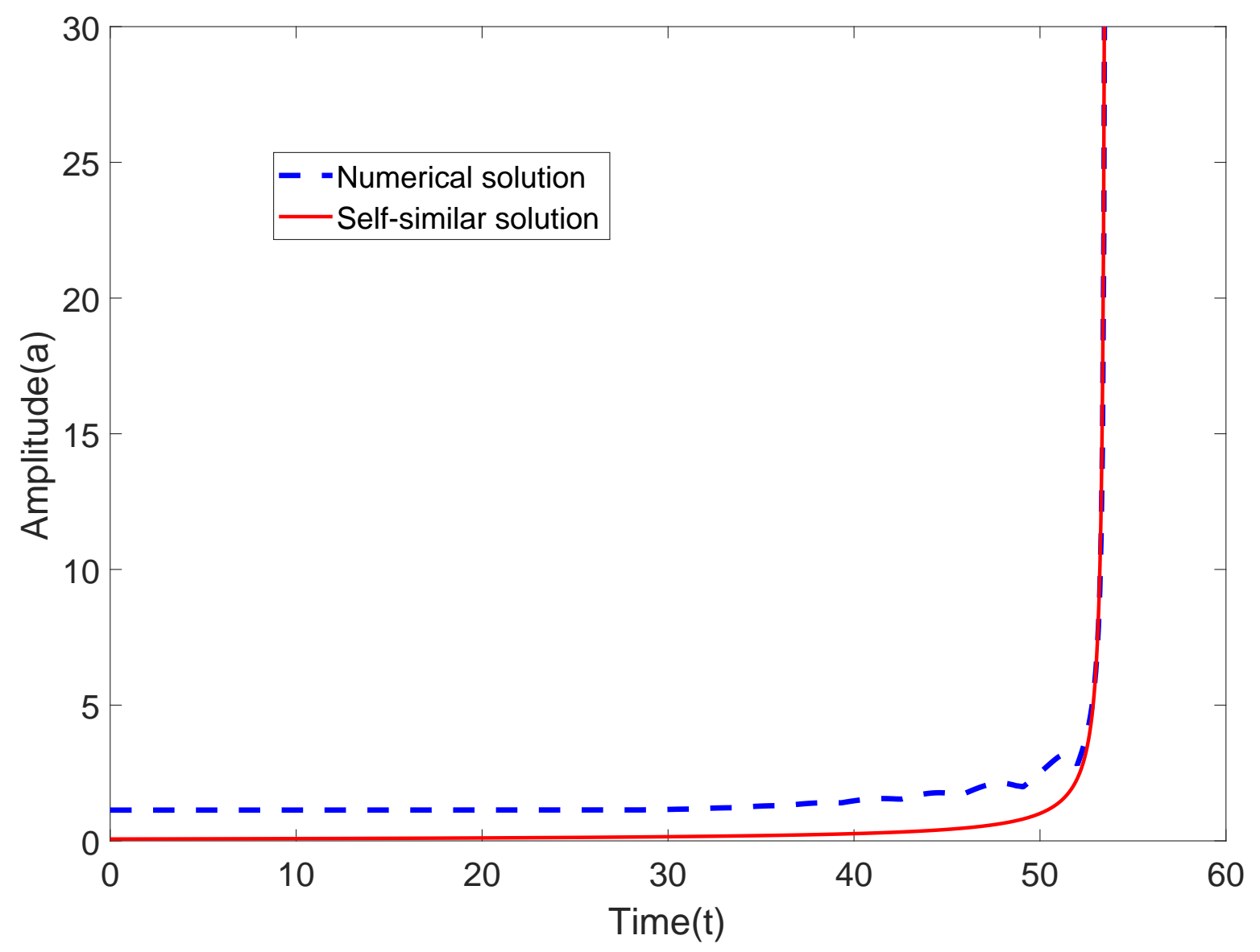

Figure 3: Example of time evolution of the amplitude of a collapsing pulse with $D<\infty$ : The Blue dashed line shows the simulated evolution of the amplitude of a collapsing pulse with the Gaussian initial condition $(a=1.1340, \sigma=25)$ for $D=4$, and the Red solid line shows the self-similar solution (14) for $D=\infty$. 


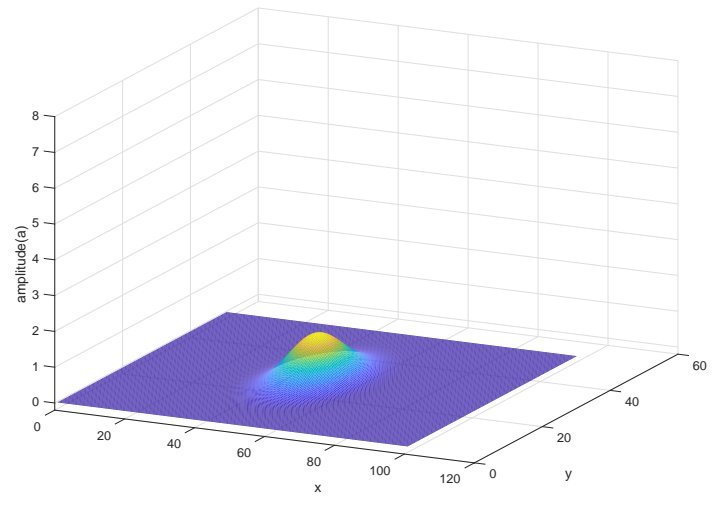

(a) $\mathrm{t}=1$

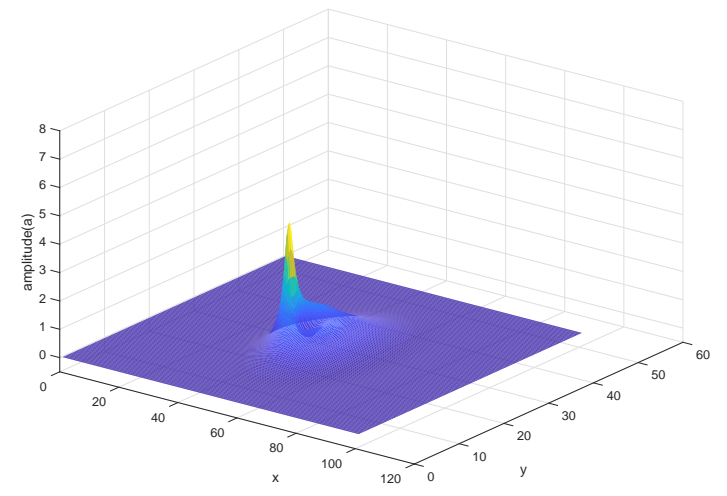

(c) $t=60$

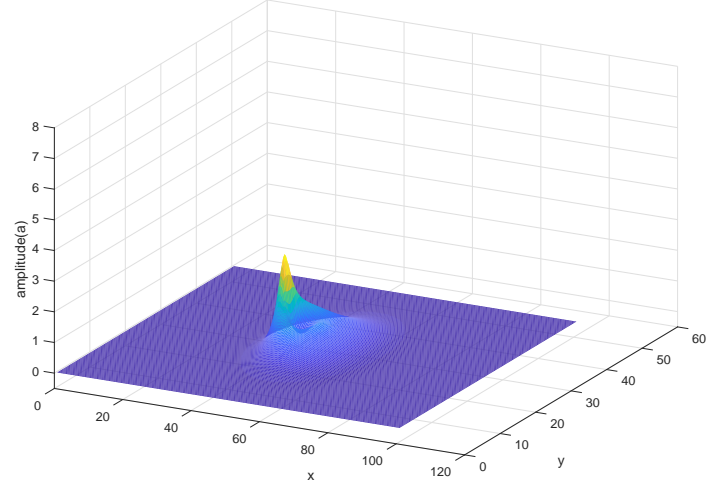

(b) $t=30$

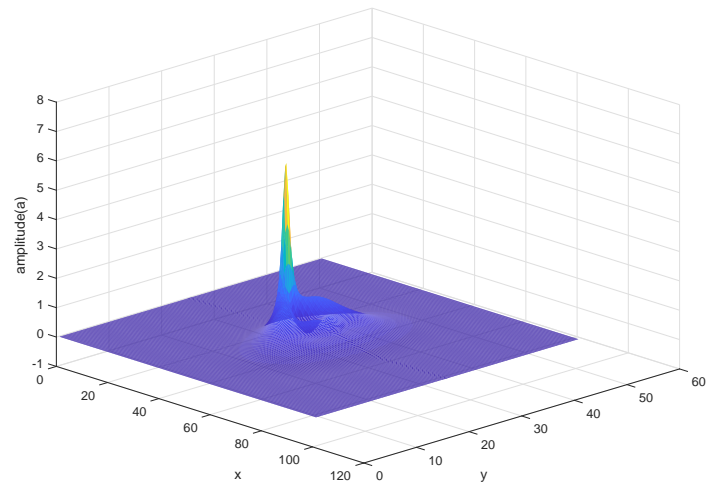

(d) $t=100$

Figure 4: Example of evolution of $A(x, y, t)$ for a collapsing pulse with the Gaussian initial distribution for the initial conditions and parameters of Fig. 3. 


\section{Concluding remarks}

Using the methodology in [10], we have shown that in the framework of the 2d-ILW equation, localized initial perturbations with negative values of the Hamiltonian $H$ collapse for all non-zero values of parameter $D$. That is, we showed that a wide class of initial conditions evolves into growing coherent patterns resembling "hairpin vortices" and "spikes" often seen in aerodynamic tunnels [26]. These patterns collapse (blow up in a finite time) in a self-similar manner, forming a point singularity.

We demonstrated this scenario by numerically simulating the 2d-ILW equation. Using the criterion $H=0$, which we verified numerically, we delineated the collapse and decay domains in the space of the parameters $a, \sigma$ and $D$. For axisymmetric Gaussian and Lorentzian initial distributions, the results were qualitatively similar and numerically close and can be summarized as follows. For large $D$, the amplitude threshold $a_{t h r}$ as a function of the horizontal scale of the initial distribution $\sigma$ is described by a hyperbola: $a_{t h r}=$ const $/ \sigma$. Any decrease of $D$ raises the threshold. For small $D$ (the 2 d-ILW equation tends to the ZK equation), the threshold stops depending on $\sigma$ and tends to infinity as $1 / D$.

At first glance, we have a large domain on the plane $(a, \sigma)$ where the initial perturbations collapse for each value of $D$, but for the collapses to be physically relevant, the applicability of the 2d-ILW equation itself, which is based on a weak-nonlinearity assumption, must be ensured. For this, the initial amplitudes of the perturbations must be sufficiently small. Obviously, the weakly nonlinear description always becomes inapplicable closer to the point of collapse, but if the initial amplitudes of collapsing perturbations are small, then we could faithfully describe a considerable part of the perturbation evolution with the 2d-ILW equation. One of our main conclusions is that physically relevant collapses are possible only for relatively large $D$ and $\sigma$ because the threshold for small values of $D$ and $\sigma$ is too high for any part of the evolution leading to collapse to be weakly nonlinear. We also recall that the derivation of the 2d-ILW equation itself breaks down for small $D$.

Our work raises a number of open questions. At present, we do not have a rigorous mathematical proof of collapses; the issue is being studied by mathematicians working on the existence of solutions of nonlinear evolution equations. The $2 \mathrm{~d}-\mathrm{ILW}$ equation that we examined is just a particular case of a more general family derived in [2]. It has now become clear how to generalize these evolution equations further to describe more complicated hydrodynamic situations (accounting for the presence of density stratification, 3d boundary layers, and a different range of Reynolds numbers), and the conditions for collapses to occur in these situations are being investigated. We note that the 2 d-ILW equation is derived for

a particular range of the Reynolds numbers: $\operatorname{Re}^{-\frac{1}{2}} \ll \varepsilon \ll \operatorname{Re}^{-\frac{1}{4}}$, where $\varepsilon$ is the nonlinearity parameter defined as the ratio of the maximum streamwise velocity of the perturbation to the maximum velocity of the basic flow. If $\mathrm{Re}=O\left(\varepsilon^{-2}\right)$, which is not rare, then the viscosity modifies the evolution equation, and a Rayleigh-type friction term is added to the $2 \mathrm{~d}-\mathrm{ILW}$ equation that we examined here. To our knowledge, there are no mathematical tools (other than direct numerical simulations) for the evolution equation with such a friction that would allow predicting the occurrence of collapses. 
The 2d-ILW equation is a weakly nonlinear asymptotic model. We do not know the eventual outcome of the found collapses in the full Navier-Stokes equations. There are several plausible options. The collapses might lead to formation of coherent strongly nonlinear patterns that might be long-lived. Most likely, the collapses cause a transition to turbulence. The role of the collapses in the bigger picture of boundary-layer instabilities and laminar-turbulent transitions remains the biggest open question. Resolving these outstanding questions will provide new insights into the physical mechanisms of boundary-layer instabilities and laminar-turbulent transitions.

\section{Acknowledgements}

The authors are grateful to the Commonwealth Scholarship Commission for the support of Joseph Oloo under grant KECA-2016-30, without which this work would not have happened. The work was also partly supported by UK NERC grant NE/M016269/1, by EU grant FP7 612610, which is gratefully acknowledged.

\section{Conflicts of Interest}

The authors declare no conflicts of interest.

\section{References}

[1] P. Schmid, D. Henningson, and D. Jankowski, "Stability and transition in shear flows. applied mathematical sciences, vol. 142," 2002.

[2] V. Voronovich, V. Shrira, and Y. A. Stepanyants, "Two-dimensional models for nonlinear vorticity waves in shear flows," Studies in Applied Mathematics, vol. 100, no. 1, pp. 1-32, 1998.

[3] R. Joseph, "Solitary waves in a finite depth fluid," Journal of Physics A: Mathematical and General, vol. 10, no. 12, p. L225, 1977.

[4] D. K. T. Kubota and L. Dobbs, "Weakly-nonlinear, long internal gravity waves in stratified fluids of finite depth," Journal of Hydronautics, vol. 12, no. 4, pp. 157-165, 1978.

[5] R. Joseph and R. Egri, "Multi-soliton solutions in a finite depth fluid," Journal of Physics A: Mathematical and General, vol. 11, no. 5, p. L97, 1978.

[6] Y. Matsuno, "Exact multi-soliton solution for nonlinear waves in a stratified fluid of finite depth," Physics Letters A, vol. 74, no. 3-4, pp. 233-235, 1979.

[7] H. Chen and Y. Lee, "Internal-wave solitons of fluids with finite depth," Physical Review Letters, vol. 43, no. 4, p. 264, 1979.

[8] V. Shrira, "On the 'sub-surface'waves of the mixed layer of the upper ocean," Trans. USSR Acad. Sci., Earth Sci. Sec, vol. 308, pp. 276-279, 1989. 
[9] V. Zakharov and E. Kuznetsov, "On three dimensional solitons," Zhurnal Eksp. Teoret. Fiz, vol. 66, pp. 594-597, 1974.

[10] A. D'yachenko and E. Kuznetsov, "Two-dimensional wave collapse in the boundary layer," Physica D: Nonlinear Phenomena, vol. 87, no. 1-4, pp. 301-313, 1995.

[11] D. E. Pelinovsky and V. I. Shrira, "Collapse transformation for self-focusing solitary waves in boundary-layer type shear flows," Physics Letters A, vol. 206, no. 3-4, pp. 195202, 1995.

[12] V. E. Zakharov and E. A. Kuznetsov, "Solitons and collapses: two evolution scenarios of nonlinear wave systems," Physics-Uspekhi, vol. 55, no. 6, p. 535, 2012.

[13] M. J. Ablowitz and H. Segur, Solitons and the inverse scattering transform, vol. 4. Siam, 1981.

[14] S. Melkonian and S. Maslowe, "Two-dimensional amplitude evolution equations for nonlinear dispersive waves on thin films," Physica D: Nonlinear Phenomena, vol. 34, no. 1-2, pp. 255-269, 1989.

[15] K. Nozaki, "Vortex solitons of drift waves and anomalous diffusion," Physical Review Letters, vol. 46, no. 3, p. 184, 1981.

[16] V. Petviashvili, "Red spot of jupiter and the drift soliton in a plasma," 1980.

[17] S. Toh, H. Iwasaki, and T. Kawahara, "Two-dimensionally localized pulses of a nonlinear equation with dissipation and dispersion," Physical Review A, vol. 40, no. 9, p. 5472, 1989.

[18] G. A. Gottwald, "The Zakharov-Kuznetsov equation as a two-dimensional model for nonlinear rossby waves," arXiv preprint nlin/0312009, 2003.

[19] D. Pelinovsky and Y. A. Stepanyants, "Self-focusing instability of nonlinear plane waves in shear flows," Journal of Experimental and Theoretical Physics, vol. 78, no. 6, pp. 883891, 1994.

[20] D. G. Gaidashev and S. K. Zhdanov, "On the transverse instability of the twodimensional Benjamin-Ono solitons," Physics of Fluids, vol. 16, no. 6, pp. 1915-1921, 2004.

[21] M. Abramowitz and I. A. Stegun, Handbook of mathematical functions: with formulas, graphs, and mathematical tables, vol. 55. Courier Corporation, 1965.

[22] E. Kuznetsov, "Stability criterion for solitons of the Zakharov-Kuznetsov-type equations," Physics Letters A, vol. 382, no. 31, pp. 2049-2051, 2018. 
[23] M. Jorge, G. Cruz-Pacheco, L. Mier-y Teran-Romero, and N. F. Smyth, "Evolution of two-dimensional lump nanosolitons for the Zakharov-Kuznetsov and electromigration equations," Chaos: An Interdisciplinary Journal of Nonlinear Science, vol. 15, no. 3, p. 037104, 2005.

[24] S. A. Orszag, "Numerical methods for the simulation of turbulence," The Physics of Fluids, vol. 12, no. 12, pp. II-250, 1969.

[25] D. A. Kopriva, Implementing spectral methods for partial differential equations: Algorithms for scientists and engineers. Springer Science \& Business Media, 2009.

[26] Y. S. Kachanov, "Physical mechanisms of laminar-boundary-layer transition," Annual review of fluid mechanics, vol. 26, no. 1, pp. 411-482, 1994. 